\title{
Una Revisión a las estrategias y actividades en clase bajo el enfoque de aprendizaje invertido
}

A review of the strategies and activities in class under the invested learning approach.

Mónica María Sinisterra Rodríguez ${ }^{1}$

Fecha de recepción: 12 de junio de 2019

Fecha de aceptación: 25 de junio de 2019

( ) 2019. Universidad de Cordoba. Este es un artículo de acceso abierto distribuido bajo los términos de la licencia de uso y distribución Creative Commons Reconocimiento 4.0 Internacional (CC BY 4.0)

\section{RESUMEN}

Superar el aburrimiento en clases y permitir una participación activa del estudiante es un objetivo educativo principal en especial en enfoques como el aprendizaje invertido (flipped classroom). Sin embargo, el éxito en la implementación de este enfoque queda supeditado al conjunto de actividades en el aula que el docente prepara previamente, las cuales deben permitir la motivación suficiente en el estudiante para que se produzca aprendizaje significativo.

A través de una estrategia pedagógica tipo concurso utilizando la aplicación Anki se muestra cómo puede mejorarse la experiencia de aprendizaje a través de una actividad que conjuga colaboración, automotivación y demostración, elementos clave para un ambiente de aprendizaje significativo.

PALABRAS CLAVE: Clase invertida, aprendizaje significativo, Anki, concurso en clase.

\begin{abstract}
Overcoming boredom in classes and allowing an active participation of the student is a primary educational objective especially as invested learning (inverted classroom). However, the success in the implementation of this approach is subordinated to the set of activities in the classroom that the teacher prepared previously, which must allow sufficient motivation in the student to produce meaningful learning.

Through a pedagogical strategy type of use use of the application Anki shows how the learning experience can be improved through an activity that combines, self-motivation and demonstration, key elements for a significant learning environment.
\end{abstract}

\section{KEYWORDS:}

Inverted class, meaningful learning, Anki, contest in classl.

${ }^{1}$ Profesora Tiempo Completo Departamento de Ciencias Económicas Universidad del Cauca. Doctora en Políticas Públicas, CIDE. Agradezco los comentarios de colegas y profesores y la retroalimentación obtenida en el Diplomado de Innovaciones Pedagógicas del proyecto Innovación Cauca, Universidad del Cauca 


\section{INTRODUCCIÓN}

La importancia de motivar al estudiante hacia el aprendizaje a través de una motivación tanto extrínseca como intrínseca radica en su capacidad para generar un aprendizaje significativo, para ello no se requiere solamente un acercamiento a situaciones de la vida real que permita el conocimiento situado o la contextualización del conocimiento sino también una mayor participación del estudiante a través de aprendizaje auto-dirigido que permita un mejor dominio del conocimiento (Bolhuis, 2003). Este planteamiento requiere reconocer la presencia de aspectos emocionales en el proceso de aprendizaje (Goleman, D. 2002), lo que exige mucho más al proceso de planeación de contenidos y didácticas de un curso.

El aprendizaje invertido o clase invertida es un enfoque pedagógico centrado en el estudiante en el cual la instrucción directa se realiza principalmente fuera del aula, para aprovechar el tiempo presencial durante la clase en desarrollar actividades de aprendizaje significativo y personalizado (Bergmann y Sams, 2012). Este enfoque tiene en cuenta que los estudiantes poseen distintos ritmos y estilos de aprendizaje y busca maximizar la experiencia durante la clase. Entre las actividades a realizar en el aula se tienen discusiones, ejercicios, pruebas, laboratorios, y proyectos o actividades para resolver un problema. Pero estas actividades estaban presentes en modelos centrados en el profesor; entonces, ¿cuáles son los elementos que se requieren para que efectivamente la clase invertida genere un aprendizaje significativo?

El boom del aprendizaje invertido ha llevado a pensar que la sola inversión de roles es el motor de un nuevo proceso de aprendizaje; sin embargo, al analizar el enfoque debe ser claro que el éxito de la implementación de la clase invertida radica en el tipo de actividades que el docente logra poner en clase, por lo cual cobra importancia qué tipo de estrategias didácticas son puestas en juego, ya que estas deben lograr mejorar la experiencia de aprendizaje y desarrollar un aprendizaje significativo en los estudiantes.

Claramente, existe un vacío en explicar la relación causal clase invertida - aprendizaje significativo. ¿Qué garantiza que el conjunto de estudiantes realicen la instrucción al exterior del aula? Bergmann y Sams (2012) parecen plantear que el uso de tecnología como videos y otro material audiovisual y digital, facilita ese proceso de instrucción, pero también hay evidencia en cursos MOOCs que en videos largos los estudiantes pierden la atención (Meléndez, Román y Pinilla, 2016; Alario-Hoyos, C. et. al. 2017). Además, existe una brecha digital bajo la cual algunos estudiantes no podrán acceder en forma plena u oportuna a ciertos contenidos digitales; y una limitación en la capacidad instruccional de video de 5 a 10 minutos. Por tanto, siempre que sea viable que el estudiante realice la instrucción al exterior del aula, es claro que es posible mejorar la experiencia de aprendizaje en el aula, pero esta hipótesis también es cierta en los modelos centrados en el profesor; entonces, ¿Cuál es el verdadero cambio que realiza el aprendizaje invertido o clase invertida?

Lo que la clase invertida logra en forma interesante es que aun cuando el estudiante no realiza una gran instrucción en el exterior (en su casa), las actividades de aprendizaje dispuestas en el aula, las interacciones generadas y en general, el ambiente de aprendizaje logrado, permiten un aprendizaje significativo . Este argumento claramente pone a prueba la existencia de una posible relación causal entre la aplicación de una clase invertida y el aprendizaje significativo. Esto es, se requieren ciertas condiciones para que la clase invertida pueda conducir a un aprendizaje significativo.

En los estudios sobre clase invertida hay un sesgo a pensar que el enfoque en sí mismo puede mejorar los resultados de aprendizaje. Este artículo tiene como objetivo mostrar que el éxito de la clase invertida radica en tres factores: Primero, actividades en clase principalmente orientadas a la tarea, como ejercicios, pruebas, laboratorios, proyectos o actividades para resolver un problema; en segundo lugar, interacciones en el aula, éstas permiten al estudiante insertarse en un ambiente de aprendizaje que le provoca un aprendizaje significativo; y en tercer lugar, retroalimentación temprana, las cuales constituyen actividades para corregir errores que realiza el profesor y los propios estudiantes durante la clase.

Diseñar las estrategias pedagógicas adecuadas para trabajar en el aula, a partir de mostrar cómo no solamente resolver los ejercicios en clase (la tarea) sino generar un ambiente de aprendizaje competitivo, que explora los aspectos emocionales, como un concurso de respuestas en clase apoyado de una herramienta tecnológica como Anki puede mejorar en forma importante la experiencia de aprendizaje y acercarnos a un aprendizaje significativo. En este artículo se presenta en forma cualitativa una experiencia de aplicación de la estrategia de clase invertida que aporta evidencia para mostrar como el mecanismo causal clase invertida - aprendizaje significativo requiere un 
eslabón fundamental: actividades centradas en la tarea con alta motivación. Sin este eslabón no es posible lograr aprendizaje significativo a partir de estrategias pedagógicas de clase invertida tal como se puede observar en la Figura 1.

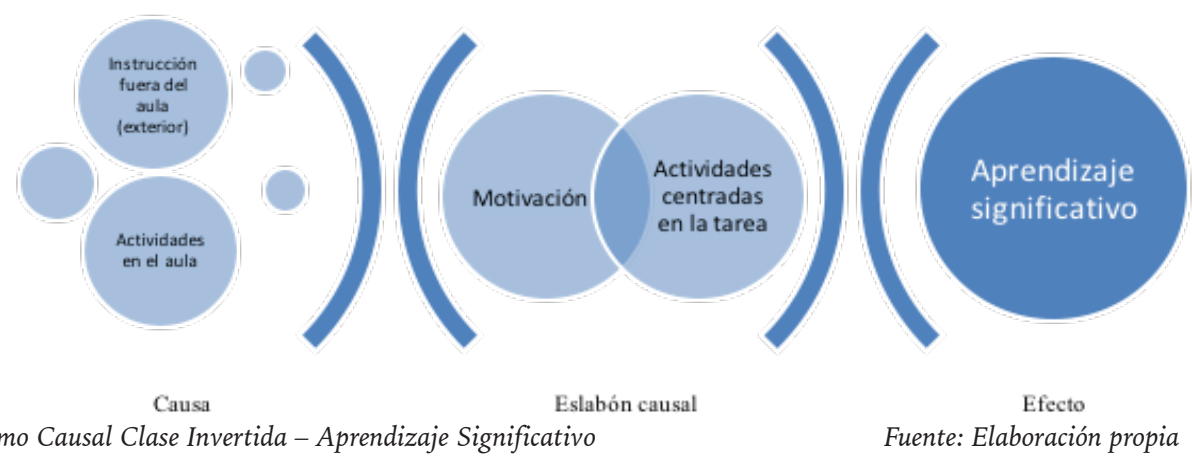

Figura 1. Mecanismo Causal Clase Invertida - Aprendizaje Significativo

Fuente: Elaboración propia

Además de esta introducción, este documento explica en la sección dos, la relación entre clase invertida y aprendizaje significativo, a partir de identificar como componente clave de la relación, el desarrollo de actividades centradas en la tarea con alta motivación. La sección tres, documenta la experiencia realizada en una clase de Microeconomía II en un curso universitario cuyos contenidos están en los niveles de la base de la taxonomía de Bloom (definir, identificar, clasificar reproducir, explicar, fundamentar), que según Bergmann y Sams (2012) constituye un nivel apropiado para la aplicación de esta metodología. Finalmente, se presentan algunas conclusiones.

\section{Actividades Centradas en la Tarea con Alta Motivación: El Eslabón entre la Clase Invertida y el Aprendizaje Significativo.}

La clase invertida busca "que lo que es realizado tradicionalmente en clase sea realizado en casa y lo que es realizado tradicionalmente como tarea sea completado en clase" (Bergmann y Sams, 2012: 13). Entre las ventajas que tiene la clase invertida se reconocen: i) los estudiantes tienen más control, ii) promueve el aprendizaje y la colaboración centrada en el estudiante, iii) los contenidos son más accesibles iv) mejor acceso (más fácil para los padres ver que está pasando) v) puede ser más eficiente al proporcionar más tiempo libre (Santiago, R., 2018). Según Bergmann y Sams (2012), la clase invertida inicia cuando al estudiante le son enviados los contenidos, muchos de ellos digitales con la explicación en video para que el estudiante la pueda revisar cuantas veces quiera, tome notas, escriba preguntas y resuma su aprendizaje. Posteriormente, al llegar a clase el estudiante presenta sus dudas, las cuales son resueltas en un lapso de tiempo muy breve y luego se les presenta la tarea o asignación del día para la cual tienen alrededor de $85 \%$ de la clase, tiempo durante el cual el docente asume un rol de tutor, y colaborador.

Para que un aprendizaje significativo se produzca se requiere generar actividades en el aula que generen alta motivación e involucramiento de los estudiantes, y que permitan aumentar el nivel de conocimiento y en este sentido, no toda discusión, ejercicio, laboratorio o proyecto cumple esta condición, muchas de estas actividades de aplicación o práctica se pueden quedar en acciones que entretienen, invitan e incluso divierten pero no acercan el conocimiento.

La estrategia pedagógica basada en actividades centradas en la tarea con alta motivación debe ser capaz de lograr que el estudiante pase de una fase de supervivencia (por ejemplo, competencia con sus compañeros), a una fase de progresión y de socialización (por ejemplo, ejercicio demostrativo en el tablero). En este sentido, los concursos en clase, se constituyen en actividades que reclaman del estudiante una necesidad básica como la de sentirse seguro, y al mismo tiempo, potencian una necesidad superior como la de reconocimiento siguiendo el esquema de necesidades de Maslowen el momento en que se realiza el ejercicio demostrativo al frente de toda la clase. Este tipo de actividad conlleva un nivel alto de inmersión que permite a los estudiantes concentrarse, se requiere también un nivel de habilidad alto al igual que un nivel de desafío superior; logrando con ello, combinar el aprendizaje activo con la creación de experiencias significativas al enlazarse 
con motivaciones intrínsecas del nivel superior como búsqueda de estatus, reconocimiento y autorrealización.

Para lograr la implementación, y posterior éxito, de una estrategia de este tipo es importante capturar cuales son las necesidades de los alumnos, para poder generar aprendizaje a través de la actividad que influencia el ambiente de aprendizaje. El ambiente de aprendizaje:

"es el contexto físico, social y pedagógico completo en el cual el aprendizaje está destinado a ocurrir... los componentes y atributos de un ambiente de aprendizaje son conceptualizados en relación a su impacto sobre el proceso de aprendizaje y los resultados en ambos dominios cognitivo y afectivo" (UNESCO, 2012: 12).

Se espera que con ambientes de aprendizaje apropiados, el aprendizaje sea efectivo, conectado y cohesivo (UNESCO, 2012). Estos ambientes se pueden construir a partir de insertar más actividades, prácticas, didácticas actualizadas, trabajo en equipo, y diversión en el aula para hacer de las clases espacios donde no abunde el aburrimiento (López y Sánchez, 2010).

Los ambientes de aprendizaje apropiados permiten aproximarse a una mejor clase, que busque una mayor cohesión y colaboración entre estudiantes, mayor involucramiento, mayor orientación a la tarea a partir de un reconocimiento de los objetivos de la clase, mejor rol del docente como apoyo; y finalmente, mayor equidad (Unesco, 2012).

Uno de los factores más importantes asociado con el aburrimiento en clase es el rol pasivo del estudiante seguido de contenidos sin significado o relación con su vida cotidiana (López y Sánchez, 2010). En este sentido, es importante que los docentes encuentren una forma de relacionarse diferente con los estudiantes y aumentar la interacción en el proceso de enseñanza aprendizaje. Yañez (2016) presenta como factores clave del proceso de aprendizaje: la motivación, el interés, la atención, la adquisición, la comprensión e interiorización, la asimilación, la aplicación, la transferencia y la evaluación; todas estas fases entrelazadas constituyen los elementos básicos para un aprendizaje significativo. La Figura 2 muestra en orden de importancia distintas formas de aprender de los estudiantes, también llamada la pirámide del aprendizaje. Este instrumento evidencia que el aprendizaje significativo se posiciona en tareas que tienen que ver con la interacción con otros, la práctica, y la discusión en grupo principalmente situados en la base de la pirámide. Mientras estrategias como las lecciones, las lecturas, y las actividades audiovisuales, tienen una menor importancia en el aprendizaje del estudiante.

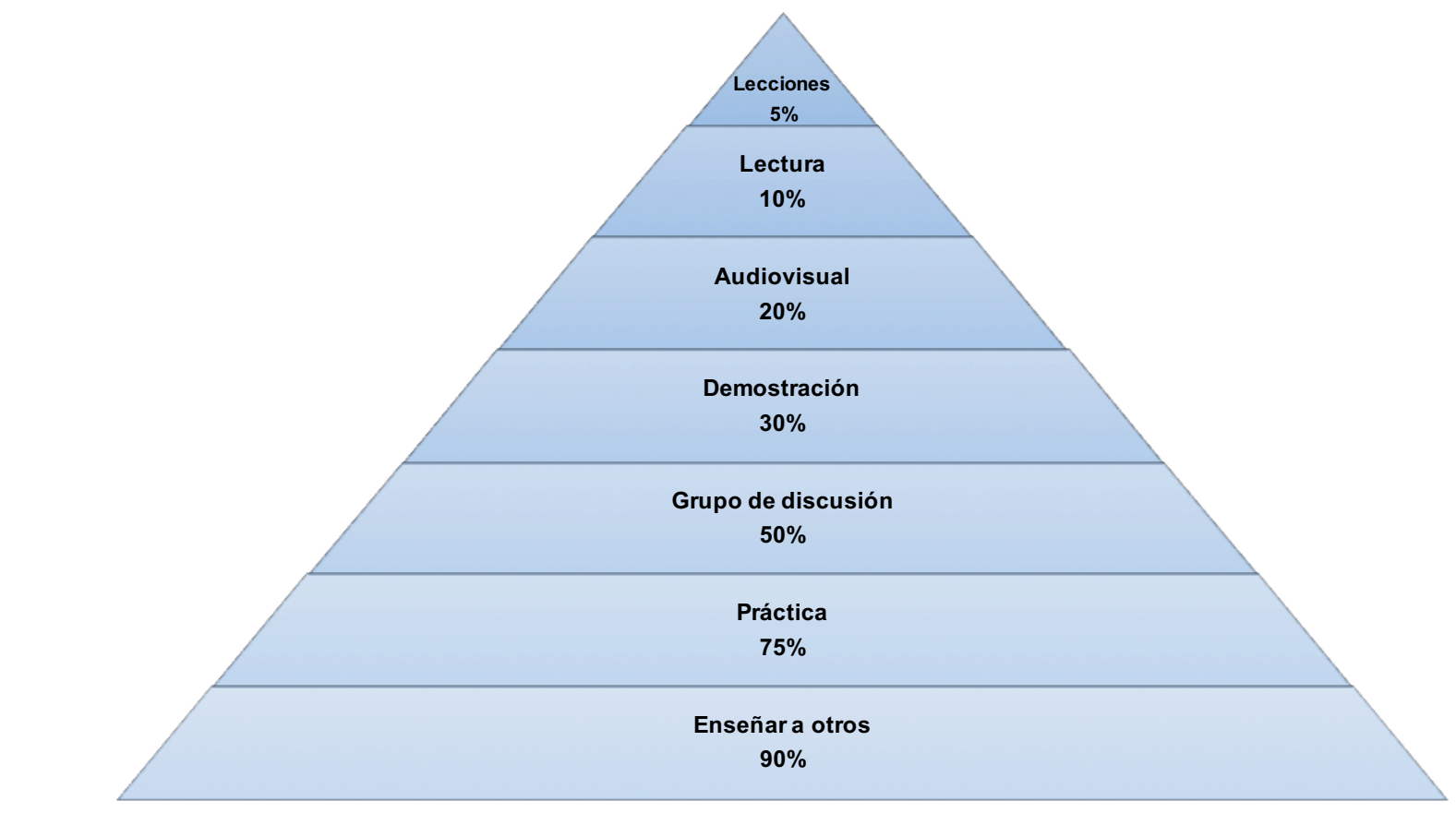




\section{Experiencia de Aplicación en Clase de una Estrategia Pedagógica para Clase Invertida}

Esta sección presenta detalles de la experiencia obtenida al aplicar clase invertida en un curso del programa de Economía de la Universidad del Cauca, inicialmente se presentan los elementos generales y posteriormente se realiza una evaluación del alcance de la estrategia en dos niveles, el primero a partir del desempeño en el curso, y el segundo a partir de la percepción de los estudiantes.

\subsection{Elementos generales de la experiencia}

La experiencia de aplicación se concentró en cuatro aspectos: la identificación del curso, la determinación de los contenidos a aprender, el diseño y presentación de la dinámica de la actividad en clase, y la descripción de la herramienta tecnológica de soporte.

Durante un curso a nivel universitario de Microeconomía II de quinto semestre con 22 estudiantes, se realizaron varias sesiones a partir del enfoque de clase invertida para desarrollar los objetivos de la tercera unidad referente a estructuras de mercado bajo competencia imperfecta. Esta unidad requiere que el estudiante, distinga los modelos de monopolio, oligopolio y competencia monopolística, del modelo de competencia perfecta estudiado en la primera unidad del curso. En este sentido, la evaluación se vuelve acumulativa en la medida que se siguen trabajando conceptos y métodos de la primera y segunda unidad, pero además es mucho más analítica porque requiere sintetizar, explicar, relacionar, predecir e interpretar y no solo, definir, describir y clasificar.

El contenido de las sesiones se distribuyó con antelación a los estudiantes y comprendía

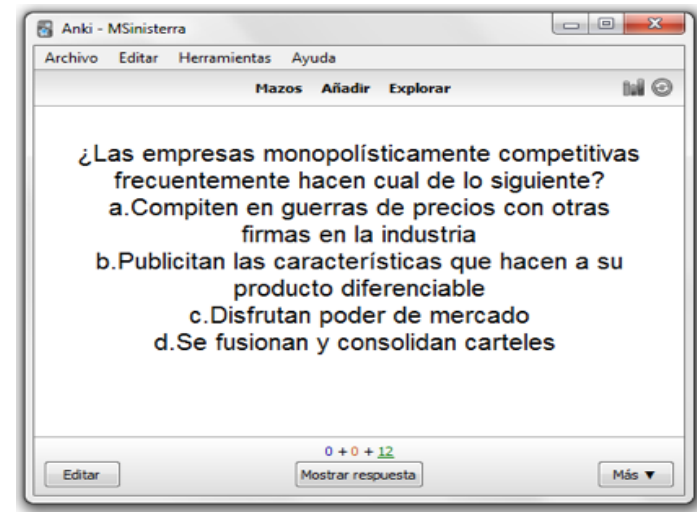

Fgura 3. . Interfaz de Anki con preguntas formuladas por la docente cuatro capítulos de texto y enlaces para ver videos explicativos cortos en Youtube que mostraban como varias industrias del entorno macroeconómico próximo, se enmarcaban en alguna de estos tipos de estructura de mercado de competencia imperfecta. Los videos tenían como objetivo contextualizar los conceptos y dar sentido a los conocimientos por desarrollar. Se solicitó a los estudiantes, no solo completar las lecturas sino también realizar los ejercicios de cada capítulo para auto evaluar su comprensión de los temas.

Al llegar a la clase, se les presentó un concurso en el cual participaban los estudiantes en grupos de tres personas, conformados por ellos mismos; se lanzaba una pregunta a toda la clase, esta pregunta se visualizaba en pantalla a través de un videobeam, el primero en levantar la mano respondía la pregunta o salía al tablero a realizar el ejercicio, los estudiantes contaban con unos minutos para colaborar con los miembros de su grupo, afinar la respuesta y salir a responderla.

A través de la aplicación gratuita Anki se presentaron las preguntas y luego la respectiva respuesta. La herramienta tecnológica operó como un apoyo en la medida que permite presentar varias preguntas; unas cortas de rápida respuesta (Imagen 1a), otras con imágenes (Imagen 1b) y otras de respuesta larga a partir de la solución de un ejercicio en el tablero. Anki es catalogada como una herramienta de productividad orientada a la memoria (EMOWE, 2018) y digitaliza la función de las antiguas fichas mnemotécnicas. Su funcionamiento básico opera a partir de una serie de mazos. En el estudio individual la herramienta tiene en cuenta los tiempos de respuesta y presenta estadísticas al usuario. El uso para la clase fue básico, y consistió en generar eficiencia y rapidez en la presentación de las preguntas y respuestas.

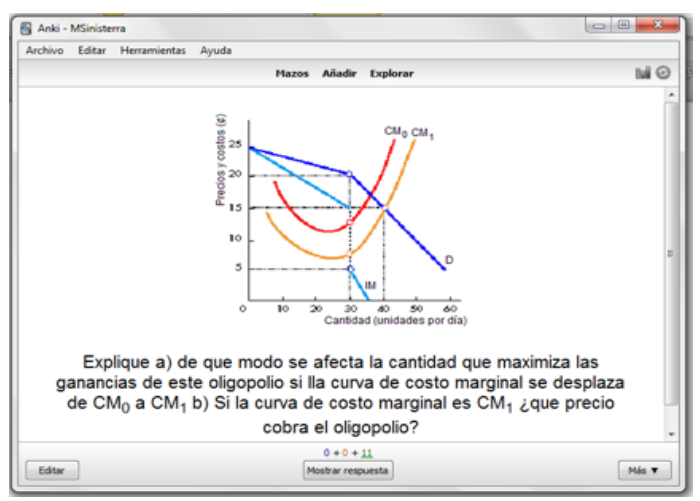


La actividad del concurso permite realizar clase invertida. Los ejercicios se realizan en clase pero premia a los estudiantes que estudiaron de mejor manera los contenidos en casa, quienes tienen la oportunidad de reforzar su aprendizaje y aumentan su compromiso con la clase y los temas. Los estudiantes que no prepararon los contenidos tienen la oportunidad de recibir ayuda por parte de sus compañeros, aprender los ejercicios básicos y de obtener una demostración de la aplicación de los mismos, interactuar con otros compañeros para aprender a realizar los ejercicios.

\subsection{Evaluación de la estrategia}

Con el objetivo de evaluar si la estrategia pedagógica fue efectiva, se tomó el promedio de las notas de las primeras evaluaciones (un parcial y un taller) realizadas antes de hacer el concurso; y el promedio de las notas de las evaluaciones realizadas después del concurso (un examen final, un quizz y un trabajo); se omitió la nota obtenida durante el concurso. La media de las notas antes del concurso fue de 3.0 y la media de las notas después del concurso fue 3.57. Para probar la hipótesis de si las diferencias son significativas antes y después del concurso, se realizó una prueba de diferencia de medias, resultando al 95\% de confianza que existe suficiente evidencia para afirmar que el efecto del concurso fue efectivo en mejorar las notas de los estudiantes (Tabla 1).

Tabla 1. Diferencia de Medias Antes y Después de la Estrategia Pedagógica

\begin{tabular}{|l|r|r|}
\hline & \multicolumn{1}{|c|}{ Variable 1 } & Variable 2 \\
\hline Media & 3.00454545 & 3.57878788 \\
\hline Varianza & 0.39735931 & 0.41762386 \\
\hline Observaciones & 22 & \\
\hline Coeficiente de correlación de Pearson & 0.11324657 & \\
\hline Diferencia hipotética de las medias & 0 & \\
\hline Grados de libertad & 21 & \\
\hline Estadístico t & -3.16827072 & \\
\hline $\mathrm{P}(\mathrm{T}<=$ t) una cola & 0.00231597 & \\
\hline Valor crítico de t (una cola) & 1.7207429 & \\
\hline $\mathrm{P}(\mathrm{T}<=$ t) dos colas & 0.00463193 & \\
\hline Valor crítico de t (dos colas) & 2.07961384 & \\
\hline
\end{tabular}

Los anteriores resultados indican que es posible atribuir una mejoría en las notas posterior a la realización de la clase invertida. Sin embargo, la tendencia al alza en las notas de los estudiantes puede deberse a otros factores, como que los estudiantes realizan un último impulso al final para no perder la materia.

Buscando hacer más robustos los resultados de la evaluación de la experiencia, se realizó ex post (al iniciar el siguiente semestre) una encuesta a todos los estudiantes que participaron en el curso, independientemente de la nota final obtenida. La encuesta se realizó en forma anónima utilizando google forms vía internet, y se obtuvo una tasa de respuesta de 64\% (14 de 22 estudiantes respondieron) que permite asumir la no respuesta como en desacuerdo con la estrategia, esto es, un $36 \%$. A la pregunta ¿considera que la estrategia 
utilizada contribuyó positivamente a su proceso de aprendizaje? el $92.9 \%$ de los estudiantes respondieron que si, y el restante $7.1 \%$ respondió que tal vez. Estos resultados corroboran por tanto, la idea de que el $64 \%$ del curso consideró la estrategia pedagógica como capaz de contribuir positivamente a su proceso de aprendizaje, y $36 \%$ no.

A la pregunta de las siguientes opciones ¿Cuál considera la estrategia utilizada contribuyó más? El

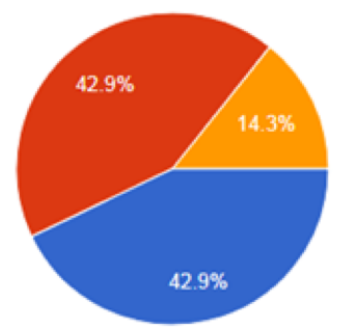

Fgura 3. . Aspectos en los que contribuyó la clase invertida

A la pregunta $4 \dot{c}$ considera conveniente utilizar estrategias de este tipo (resolver los ejercicios a manera de concurso en el tablero) para el aprendizaje en sus asignaturas? el $71,4 \%$ respondió que si, el $14,3 \%$ respondió que no, el $7,1 \%$ si, pero con competencia entre subgrupos, y el restante respondió que la estrategia es conveniente dependiendo del tipo de asignatura. Esto muestra que entre los estudiantes que consideraron valiosa la estrategia, la mayoría (más del $70 \%$ ), considera que el concurso es una actividad apropiada para Tabla 2. Objetivos de la Estrategia Pedagógica

\begin{tabular}{|c|c|c|c|}
\hline objetivos & Freq. & Percent & Cum. \\
\hline aprenderloscontenidos & 4 & 28.57 & 28.57 \\
\hline evaluar & 1 & 7.14 & 35.71 \\
\hline fomentarcompetencia & 1 & 7.14 & 42.86 \\
\hline fomentarparticipacion & 3 & 21.43 & 64.29 \\
\hline motivar & 4 & 28.57 & 92.86 \\
\hline estudiar & 1 & 7.14 & 100.00 \\
\hline Total & 14 & 100.00 & \\
\hline
\end{tabular}

Finalmente, se buscó mostrar la existencia de una relación entre los objetivos que reconocieron los
$42,9 \%$ respondió generar motivación por estudiar y comprender, el $42.9 \%$ generar ejemplos de solución de los ejercicios y el $14,3 \%$ generar competencia entre compañeros y compañeras. Este resultado es importante porque se conecta claramente con la teoría, uno de los mayores objetivos del aula invertida es aumentar la motivación del estudiante por estudiar y comprender seguido del aprendizaje de un contenido específico (Figura 3).

Fuente: Elaboración propia

aprender. En el total de estudiantes que recibió la clase esto representa el $45.45 \%$.

Se preguntó también con que objetivos se realizó la estrategia pedagógica, y los estudiantes reconocieron como objetivos principales: aprender los contenidos y motivar ( $28.57 \%$ cada uno). Además, establecieron como otros objetivos de la estrategia, fomentar la participación $(21,43 \%)$, evaluar $(7,14 \%)$, fomentar la competencia $(7,14 \%)$ y estudiar $(7,14 \%)$, tal como se observa en la tabla 2.

estudiantes satisfacía la estrategia y el principal aporte de la misma, encontrando resultados interesantes, tal como se puede observar en la 


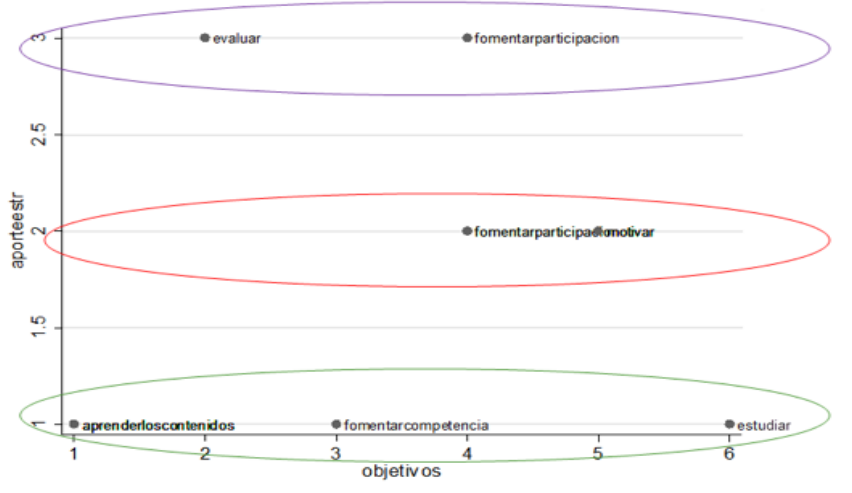

Figura 4. Relación entre Aporte de la estrategia pedagógica y sus objetivos Fuente: Elaboración propia

figura 4.

Los estudiantes que percibieron que la estrategia contribuía a generar ejemplos de solución de los ejercicios también consideraron que sus objetivos eran aprender los contenidos, fomentar la competencia y estudiar. Los estudiantes que percibieron que la estrategia contribuía a generar motivación también consideraron que sus objetivos eran fomentar la participación y motivar; y finalmente, estudiantes que percibieron que la estrategia contribuía a generar competencia consideraron que sus objetivos eran evaluar y fomentar la participación. Esta relación es muy importante porque evidencia la presencia de tres personalidades o tipos de estudiantes, al interior de la clase.

También se relacionó la contribución de la estrategia con su conveniencia, y se encontró al $95 \%$ de confianza, que en general el $76,92 \%$ de los estudiantes que creen que es conveniente también considera que les contribuye en su aprendizaje. Este resultado apuntala la importancia de dejar claros en los estudiantes los objetivos de aprendizaje al aplicar cualquier estrategia pedagógica, si los estudiantes la consideran valiosa, así mismo la consideraran apropiada, y viceversa (Tabla 3 ).

Tabla 3. Relación entre contribución y conveniencia de la estrategia pedagógica

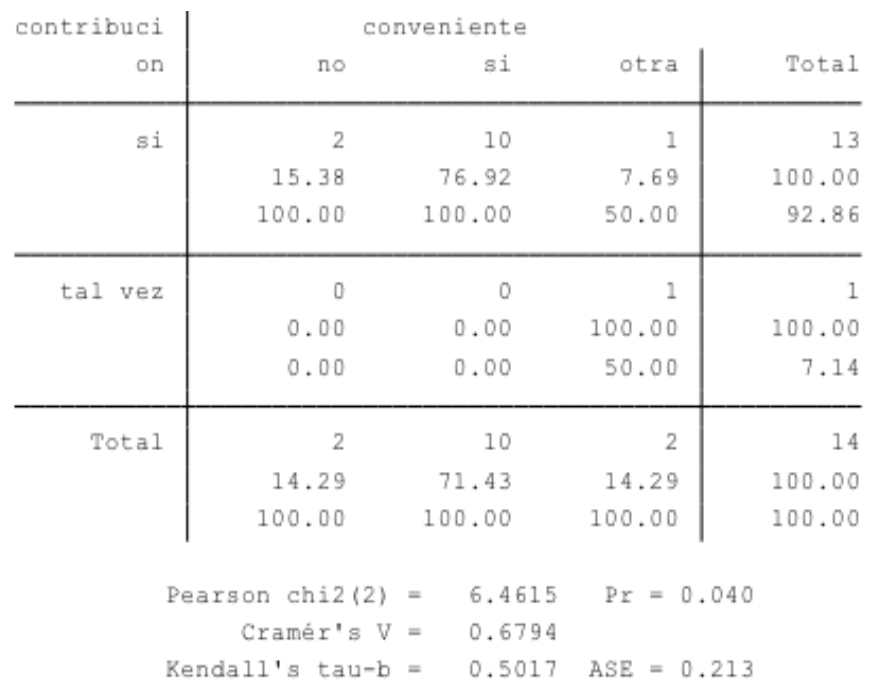

Finalmente, se consultó a los estudiantes por los aspectos a mejorar, se realizó una pregunta abierta sin opciones para lograr captar todos los factores que los estudiantes percibieron como claves en el desarrollo de las actividades en la clase invertida
(Tabla 4). El 35,71 consideró que es necesario más tiempo, el 14,29\% respondió más incentivos y el restante se difumina en un conjunto de respuestas entre las que se encuentran: que todos ganen, más grupal, más organización, más teoría, 
más discusión, más ejemplos reales, e identificar diferencias en temor a salir al tablero. Entre esta variedad de respuestas es de destacar la más formulada: más tiempo. Este es uno de los mayores problemas de la clase invertida, se requiere más tiempo para completar las actividades en clase.

Tabla 4. Aspectos de la estrategia mejora

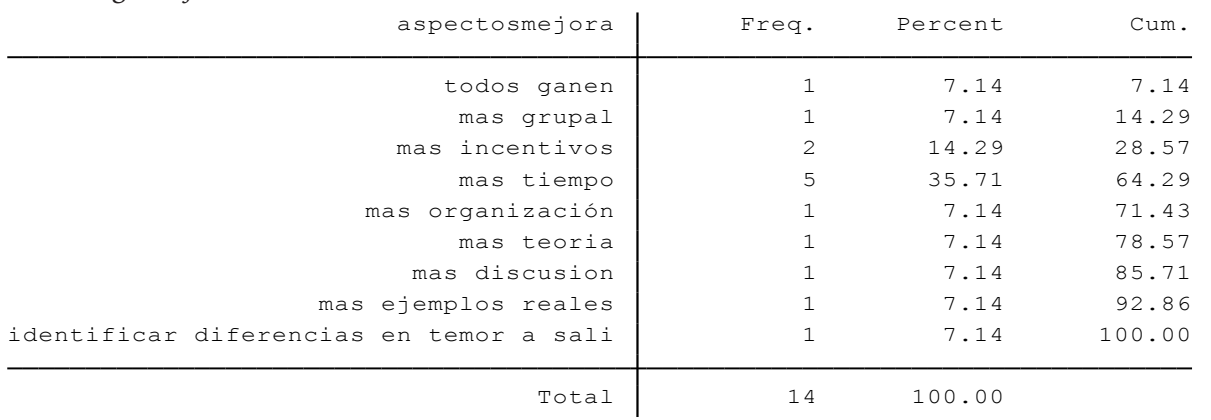

El reconocimiento del éxito de un alumno o de un grupo de alumnos los motiva a mejorar y seguir aprendiendo, ya que este reconocimiento es público e inmediato, los estudiantes se preparan para la siguiente ronda de preguntas. El conocer las causas del éxito o del fracaso hace parte fundamental de la actividad y aumenta la motivación intrínseca, esta que viene impulsada por su necesidad de reconocimiento y autorrealización. El sentimiento de competencia actúa como factor de motivación en los estudiantes, le permite sentirse competente, con capacidad de aprender y superar desafíos, "lo que favorece que tenga sentido realizar el esfuerzo necesario para conseguirlo" (Carrillo et. al., 2009: 29).

Finalmente, una de las ventajas del uso de Anki, es que se reivindica la memoria dado que las estrategias de aprendizaje activo, y centradas en el alumno no van en contravía de los procesos de memoria. La memoria actúa como depositaria de todo aprendizaje y permite aprender más y mejor, esto es llegar a niveles más profundos o de mayor complejidad en el conocimiento. Más aún, la memoria se apoya en hábitos de aprendizaje para investigar, indagar, descubrir y crear conocimiento, hábitos que contribuyen a la formación del estudiante en distintos aspectos de la vida.

La combinación de este instrumento con la experiencia emocional que trae consigo a la clase el concurso, permiten generar un aprendizaje significativo. La actividad de aprendizaje (el concurso de preguntas), y la tecnología educativa (Anki) buscan que el estudiante deje su rol pasivo en el que no hace nada y cambie a atender la clase activamente, a resolver problemas durante la clase, a colaborar con su grupo de tal forma que no se siente solo y está ocupado. Por su parte, el maestro direcciona y corrige los ejercicios, permite que los estudiantes participen, así la clase no gira en torno a él, y se logra una mayor explicación de los problemas.

\section{Discusión y conclusiones}

La labor pedagógica del docente enfrenta la necesidad de llegar al estudiante de manera innovadora, capturando su atención y facilitando el aprendizaje. La clase invertida es un enfoque innovador e interesante que puede contribuir a generar aprendizaje significativo. En este artículo hemos encontrado que para que ese resultado ocurra es necesario que las actividades que se realizan en la clase invertida estén orientadas a la tarea y tengan alta motivación, cuestión que puede lograrse con estrategias pedagógicas del tipo concurso en clase, el cual permite elevar la motivación intrínseca de los estudiantes y aumentar el conocimiento de los contenidos de clase.

Para que el aprendizaje significativo ocurra es necesario que las actividades que se realizan en la clase invertida estén orientadas a la tarea y tengan alta motivación. El uso de tecnologías a manera de apoyo es relevante; la tecnología actúa como instrumento que permite adoptar y compartir nuevas experiencias, además que hace más eficientes ciertas tareas; sin embargo, la tecnología no debe usarse para entretener a los estudiantes a manera de hacer más dinámica la clase o buscando "deslumbrar" a los estudiantes con las herramientas más recientes disponibles. La tecnología debe usarse para que los estudiantes apropien contenido y aprendan más y mejor. Anki ayuda a memorizar y comprender conceptos a través de nuevos caminos, hace la rápida la interacción pregunta - respuesta, y permite colaborar compartiendo los contenidos de los flashcards o mazos; sin interrumpir en lo 
verdaderamente valioso de la clase invertida, que son los estudiantes trabajando en clase su proceso de construcción de conocimiento acompañados. La estrategia pedagógica presentada puede ser considerada una innovación educativa en la medida que es capaz de generar verdaderas mejoras en el proceso de aprendizaje, pero es necesario realizar más estudios con tamaños de muestra más grande para aumentar la confiabilidad de este resultado. La innovación educativa "exige una condición positiva, al menos en teoría. Puede que luego resulte un fracaso el desarrollo de esa innovación, pero el núcleo de su incorporación está en la expectativa de mejora" (Santos, M. 2017: 2). En este sentido, estudios futuros pueden centrarse en la comparación cualitativa de los resultados de la clase invertida mostrando a través de diseños experimentales (con grupo de control y de tratamiento) evidencias de diferencias en el tipo de actividad desarrollada y en el tipo de estudiantes para aportar a soportar el mecanismo causal aquí formulado.

El $36 \%$ de no respuesta en los encuestados, puede entenderse como la no conformidad con la estrategia pedagógica. Además, se encontró que la mayor limitación de la estrategia de clase invertida es el tiempo. Estos hallazgos hacen necesario para los docentes interesados en implementar este tipo de estrategias pedagógicas, invertir más tiempo previo en identificar el tipo de estudiante con el que interactúa, así como, las fases durante las clases.

Las personas que consideraron que el principal objetivo de la clase invertida fue el aprendizaje de contenidos también vieron en este el principal aporte. Por lo que el establecimiento de los objetivos de aprendizaje es fundamental para lograr un mayor alcance y aproximarnos a un aprendizaje significativo en los estudiantes.

\section{REFERENCIAS}

[1]. Alario-Hoyos, C., Estevez-Ayres, I., Delgado, C. y Villena-Román, J. (2017). From MOOCs to SPOCs... and from SPOCs to Flipped Classroom. En EC-TEL 2017. Springer, pp. $347-354$.

[2]. Bergmann, J., \& Sams, A. (2012). Flip your classroom: reach every student in every class every day. Retrieved from https://www.iste. org/resources/product? ID = 2285
[3]. Bolhuis, Sanneke (2003). Towards processoriented teaching for selfdirected lifelong learning: a multidimensional perspective. Learning and Instruction, 13, 327-347.

[4]. Carrillo, M., Padilla, J., Rosero, T., y Villagómez, M.S. (2009). La motivación y el aprendizaje. Alteridad. Revista de Educación, 4(2), julio-diciembre, 20-32.

[5]. EMOWE. (2018). Anki: la mejor herramienta de productividad orientada a la memoria. Disponible en: https://emowe. com/anki-memoria-productividad/

[6]. Goleman, Daniel. (2002) Inteligencia Emocional. Editorial Kairos.

[7]. López, Norma y Sánchez, Ludivina (2010) El Aburrimiento en Clases. Procesos Psicológicos y Sociales, Vol. 6 \# 1

[8]. Melendez, Alejandra, Tomán, Mariela y Pinillos, Rossana (2016). Guía práctica: gestión, producción, infraestructura y control de calidad para MOOC. en Sánchez, J. (2016) Editor. Nuevas Ideas en Informática Educativa, Volumen 12, p. 372 - 377.

[9]. Santiago, Raul (2018). Lo que deberías saber antes de despegar con el modelo Flipped Classroom. Flipped Classroom Newsletter \# 13, 16p.

[10]. Santos. M.A. (2017). Innovar o morir. http://eldiariodelaeducacion.com/blog/ author/m-a-santos-guerra/

[11]. UNESCO. (2012). A Place to Learn: Lessons From Research on Learning Environments. UNESCO:Paris. 89p.http://unesdoc.unesco. org/images/0021/002154/215468e.pdf

[12]. Yañez, Patricio (2016). El Proceso de Aprendizaje: Fases y Elementos Fundamentales, Revista San Gregorio, 1(11), $70-82$. 